\title{
STELLUNGNAHME
}

\section{Antwort der Autoren zum Leserbrief von D.M. Ringbeck}

$\mathrm{W}$ ir danken dem Kollegen Ringbeck für die eingehende Darstellung der Bewertung myokardialer Pathologien mit Hilfe der kardialen Magnetresonanztomographie (MRT) im Kontext ischämischer Ereignisse, die im Rahmen der Fallvorstellung nicht im Fokus der Diskussion standen.

Die Pathophysiologie der $\mathrm{Ta}-$ ko-Tsubo-Kardiomyopathie ist weitgehend ungeklärt. Entsprechend komplex erscheint die Wertung der beschriebene MRT-Phänomene bei dieser Entität. Einerseits impliziert dies, dass, basierend auf den Erfahrungen mit akuten Infarkten und Myokardnarben, die zu erwartenden MRT-Bildphänomene bei Tako-Tsubo-Kardiomyopathie schwierig vorauszusagen, andererseits die vorliegenden Beobachtungen ebenso komplex in ihrer Interpretation sind. Im vorliegenden Fall wurde ein nur transientes ,late gadolinium enhancement" (LGE) beschrieben, was dem initialen, der Infarktbetrachtung entstammenden ,,bright is dead"-Paradigma widerspricht. Offen- sichtlich basiert das hier beschriebene LGE - zumindest zum Teil - auf einem myokardialen Ödem und dem damit zusammenhängenden vergrößerten Verteilungsvolumen für die extrazellulären Gd-(Gadolinium-)Kontrastmittel (,pooling“"). Dieses Ödem und der entsprechende Rückgang im kurzfristigen Verlauf wurden durch die T2-gewichteten Turbospinechosequenzen bewiesen. Somit ist auch mit einem Rückgang des LGE zu rechnen. Als weiterer Kontributor für die LGE-Entstehung kommt auch eine diffuse Verteilung von nekrotischen Kardiomyozyten in Betracht, welche im kurzfristigen Verlauf zu einer unterschwelligen, visuell nicht mehr detektierbaren Kontrastmittelanreicherung führen kann. Eine quantitative Gewichtung von Ödem und Nekrose ist daher gerade im Fall der Tako-Tsubo-Kardiomyopathie nicht möglich.

Abschließend möchten wir noch darauf hinweisen, dass der Terminus „late gadolinium enhancement" einzig das Zeitverhältnis zwischen Kontrast- mittelapplikation und Bildakquisition beschreibt und nicht auf das Stadium der myokardialen Pathologie abhebt. In diesem Zusammenhang stellt die Bestimmung des ,,relative enhancement" in Abgrenzung zur First-Pass-Perfusion lediglich eine quantitative Spielart des LGE dar, die nicht visuell identifizierbare Kontrastmittelanreicherungen erfassen soll und unabhängig vor der verwendeten Sequenztechnik (Spinecho vs. Gradientenecho) ist.

\section{Korrespondenzanschrift}

Priv.-Doz. Dr. Peter Hunold

Leitender Oberarzt

Klinik für Radiologie und

Nuklearmedizin

Universitätsklinikum Schleswig-Holstein

Campus Lübeck

Ratzeburger Allee 160

23538 Lübeck

Telefon (49/451) 500-2129

Fax -3060

E-Mail: peter.hunold@uk-sh.de 